\title{
Lidil
}

Revue de linguistique et de didactique des langues

$51 \mid 2015$

Acquisition du langage et phonologie

\section{Apprendre le français avant l'anglais au Liban : un ordre en questions}

Learning French before English in Lebanon: A Sequence in Question

Jacqueline Billiez et Carla Serhan

\section{(2) OpenEdition}

Journals

Édition électronique

URL : http://journals.openedition.org/lidil/3763

DOI : 10.4000/lidil.3763

ISSN : 1960-6052

Éditeur

UGA Éditions/Université Grenoble Alpes

Édition imprimée

Date de publication : 30 mai 2015

Pagination : 201-216

ISBN : 978-2-84310-297-4

ISSN : $1146-6480$

Référence électronique

Jacqueline Billiez et Carla Serhan, « Apprendre le français avant l'anglais au Liban : un ordre en questions », Lidil [En ligne], 51 | 2015, mis en ligne le 30 novembre 2016, consulté le 19 avril 2019. URL : http://journals.openedition.org/lidil/3763 ; DOI : 10.4000/lidil.3763

(c) Lidil 


\title{
Apprendre le français avant l'anglais au Liban : un ordre en questions
}

\author{
Jacqueline Billiez* et Carla Serhan**
}

\begin{abstract}
RÉSUMÉ
Cet article vise à interroger un postulat portant sur l'enseignement des langues au Liban et plus précisément sur une représentation majoritaire qui consiste à concevoir l'enseignement/apprentissage du français, langue prétendument difficile, avant l'anglais, langue prétendument facile, comme garant du trilinguisme libanais. Cette représentation sociale des langues et de leur ordre d'apprentissage, prépondérante chez les enseignants ainsi que les spécialistes de ces langues, limite les étudiants dans leurs choix de cursus universitaires au point que rares sont ceux qui, sortant d'écoles «anglophones», osent se lancer dans une filière où le français serait requis. Deux entretiens menés auprès d'étudiants ayant réalisé ce parcours «à revers» visent à illustrer que ce dernier n'est pas impossible à réussir.
\end{abstract}

\begin{abstract}
This article questions a postulation about language teaching in Lebanon that consists in conceiving a sequence in language teaching/learning process: French should be taught before English because French language is considered hard to learn while English language is easy. This major representation of languages and their teaching is widely spread between teachers and language specialists and therefore limits university students' academic choices, since very few of Anglophone students dare to enroll a department where French is required. Two interviews done with students who learned French language after English are developed in this paper in order to illustrate that this learning sequence is always possible.
\end{abstract}

* Univ. Grenoble Alpes, LIDILEM.

** Université de Balamand, Liban. 


\section{Introduction}

À l'heure actuelle, au Liban, deux langues sont en situation de compétition dans le système éducatif qui prévoit leur enseignement précoce, en plus de celui de la langue arabe. Les élèves libanais se trouvent majoritairement scolarisés dans des écoles dites «francophones» où ils apprennent l'anglais en troisième langue (Haddad, 2002; Hoyek, 2004; Hoteit, 2010). Tandis que d'autres, actuellement minoritaires, sont scolarisés dans des écoles où le médium d'enseignement est l'anglais, le français n'intervenant qu'à la troisième place comme langue enseignée. Si ces derniers, devenus étudiants, se dirigent quasi naturellement vers les universités anglophones, ceux sortis des écoles «francophones» eux aussi se tournent de plus en plus vers les universités «anglophones» où le français n'a plus voix au chapitre. Si les élèves scolarisés dans des écoles libanaises «francophones» arrivent à intégrer les universités «anglophones», ceux scolarisés dans des écoles «anglophones» considèrent d'office qu'ils n'arriveront jamais à faire leurs études supérieures en français, alors qu'ils ont appris cette langue comme troisième langue à l'école. Ils suivent en cela une représentation sociale, très largement partagée au Liban, qui prône un ordre d'apprentissage allant du français, langue réputée difficile, vers l'anglais, langue considérée comme facile. Dans cette contribution, nous remettons en cause cette représentation pour tenter de donner aux étudiants «anglophones» la possibilité d'être trilingues au même titre que leurs homologues «francophones» et, éventuellement, de faire des études supérieures en français. Avec le dessein de favoriser, lors de formations initiale et continue, la prise de conscience de ces représentations par les enseignants, nous analysons des entretiens semi-directifs menés auprès de deux étudiants, très minoritaires, qui ont osé effectuer le parcours inverse de cet ordre. Ce travail à partir de ces représentations peut, selon nous, aider à la rationalisation de cette hiérarchisation chronologique dans l'apprentissage des langues au Liban.

\section{Le postulat de l'ordre dans les apprentissages des langues au Liban}

\subsection{La situation majoritaire}

Les Libanais, qu'ils soient spécialistes des langues ou profanes, avancent comme un postulat que l'apprentissage de la langue française avant celui de la langue anglaise facilite l'apprentissage de cette dernière. À 
tout parent qui désire que ses enfants soient trilingues, on conseille, dès l'école maternelle, un établissement «francophone» qui enseigne, outre l'arabe littéral, d'abord le français comme langue seconde (donc langue outil ou medium d'enseignement) et ensuite l'anglais comme troisième langue. Selon la sociolinguiste Hoteit (2010), «les parents ont tendance à orienter leurs enfants vers une école francophone au début de leur scolarisation afin d'acquérir un bon bagage linguistique et culturel en français, lequel faciliterait plus tard l'apprentissage de l'anglais » (p. 53).

Ce chemin est tellement bien suivi qu'il s'ensuit la représentation dominante d'un trilinguisme qui ne serait l'apanage que des seuls francophones, «c'est-à-dire de ceux qui ont appris le français comme première langue étrangère et qui ont appris les disciplines scientifiques en français » (Hoyek, 2004, p. 49). Mais quelles raisons donne-t-on à l'adoption de cet ordre dans l'apprentissage des langues? Le motif est toujours relié à la prétendue difficulté de la langue française en comparaison avec la prétendue facilité de la langue anglaise (Haddad, 2002). Ces représentations épistémiques du français et de l'anglais circulentelles dans d'autres zones géographiques? A-t-il jamais été prouvé que l'apprentissage du français doive précéder celui de l'anglais puisqu'il le faciliterait?

Quelles que soient les réponses à ces questions, la réalité de la situation libanaise est qu'au moment du choix de l'université à intégrer, les étudiants «francophones » (c'est-à-dire ceux qui ont suivi leur cursus entier avec le français comme langue d'enseignement) se dirigent de plus en plus vers les universités «anglophones » (Chéhadé, 2008; Hafez, 2006; Diab, 2006 ; Smailly-Hajjar, 1996), alors qu'il est rarissime qu'un étudiant «anglophone»s'inscrive dans une université «francophone» ou dans un département de français faisant partie d'une université majoritairement «anglophone» : «inutile de rappeler que les étudiants francophones sont en mesure de suivre un cursus anglophone alors que le cas inverse est rare» (Hafez, 2006, p. 125).

Remettre en cause ces représentations des langues et de leur apprentissage permettrait sans doute de renverser la donne en faisant entrevoir aux étudiants «anglophones » la possibilité de faire des études universitaires entièrement ou partiellement en français.

\subsection{Deux cas atypiques}

Pour essayer de mieux comprendre la situation et donner un début de réponse à ces questions, nous avons procédé à une pré-enquête qualita- 
tive qui a consisté à réaliser deux entretiens semi-directifs de recherche auprès de deux étudiants considérés comme très atypiques au Liban. Il s'agit de Widad, libanaise et de Tarek, syrien (désormais W. et T.), qui ont fait le chemin «à l'envers» : ayant suivi un cursus de base en anglais, ils ont appris le français ultérieurement comme troisième langue. L'examen de ces deux cas ne prétend aucunement tirer des conclusions en les généralisant sur l'ensemble de la population libanaise, mais vise à illustrer avec ces deux étudiants, rarissimes mais bien réels, la possibilité offerte aux étudiants libanais de sortir des sentiers battus en réussissant à devenir trilingues tout en ayant suivi une éducation de base en anglais. Il s'agit là d'analyser leurs propos, recueillis à l'issue heureuse de leur itinéraire, et de les exploiter à des fins de formation des enseignants de langues, au premier rang desquels se trouvent ceux de français.

Au moment de se présenter au département trilingue de Langues vivantes et traduction de l'université de Balamand, W. et T. se sont heurtés à la question de la maitrise de la langue française, condition nécessaire à l'accès de ce département trilingue (arabe-français-anglais). Convaincus de leur choix de spécialisation, ils ont retardé d'une année l'intégration de ce département pour la consacrer à l'apprentissage intensif du français. C'est ainsi qu'ils ont pu atteindre le niveau B2 selon le Cadre européen commun de référence pour les langues (CECRL), niveau qu'atteint d'habitude un apprenant libanais appliqué après une quinzaine d'années d'apprentissage du français dans les écoles «francophones », et ce à raison de 6h/semaine en moyenne, sans compter les heures accordées aux disciplines non linguistiques enseignées elles aussi en français dans ces écoles (Assaf Khoury, 1998; Haddad, 2002; Hafez, 2006). En accomplissant cette «prouesse», W. et T. ont infirmé l'idée reçue et très répandue qui consiste à présenter l'apprentissage du français après l'anglais, comme mission très difficile voire impossible.

\section{Des représentations des langues française et anglaise au fondement du postulat}

Dans son enquête effectuée en 2004 auprès de 284 étudiants affiliés à

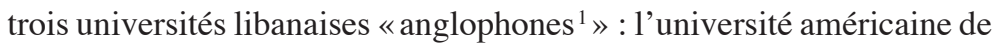

1. Les apprenants libanais ont une langue première hybride : le libanais appris en famille et pratiqué au quotidien et l'arabe littéral appris à l'école. Une 
Beyrouth, l'Université libano américaine et une université majoritairement «anglophone», l'université de Balamand, Diab (2006) a constaté que $« 81 \%$ des étudiants enquêtés [ $43 \%$ d'entre eux avaient été affiliés à des écoles francophones] ont déclaré qu'il était plus facile d'apprendre le français avant l'anglais et non le contraire» (p. 89). Cette idée se trouve parfaitement illustrée par les propos de l'un des enquêtés de Gueunier, Haddad et Aucagne (1993), dans leur livre Le français au Liban: «Une fois qu'on sait le français, c'est facile d'apprendre l'anglais» (p. 169), ou par ceux d'un autre enquêté de Hafez (2006) : «C'est beaucoup plus facile d'apprendre l'anglais quand on possède la langue française» (p. 190). Cette prétendue vertu de la langue française trouve son origine, d'après Gueunier et coll. (1993), dans les supposées facilité de l'anglais et difficulté du français. Ces données ont une influence directe sur les choix dans l'ordre de l'apprentissage de ces deux langues dans la société libanaise (Haddad, 2002).

\subsection{Le français, langue «difficile »; l'anglais, langue «facile»}

Dans l'enquête précédemment citée, Diab (2006) affirme que «les étudiants enquêtés perçoivent l'anglais comme une langue facile, voire

situation de diglossie caractérise l'une des facettes de la pratique linguistique, ce qui est d'ailleurs le cas de tout arabophone (Abou, 1994, p. 421). Quant à la langue seconde (Cuq, Davin-Chnane, 2007), c'est la langue française pour près de $66 \%$ des Libanais, d'après les données de l'ambassade de France au Liban. Le français est appris non seulement en tant que langue, mais aussi comme véhicule des disciplines non linguistiques dans les écoles appelées «francophones» (Haddad, 2002). Après cinq à sept ans d'apprentissage des deux langues arabe et française, une troisième langue est introduite dans le cursus scolaire de ces apprenants, il s'agit de l'anglais. Le schéma inverse est suivi dans les écoles libanaises dites «anglophones» où c'est l'anglais qui est appris en tant que langue seconde, alors que c'est le français qui est introduit en troisième lieu. Dans une perspective plurilingue, ces catégories sont toutes à remettre en question puisque certains enfants libanais entrent à l'école maternelle et primaire avec des répertoires composites qui comportent déjà le français. Les guillemets sont également nécessaires lorsque nous parlons des établissements anglophones ou francophones, car il s'agit d'une ambigüité sur le statut des langues à la fois dans la conception de l'ordre des langues et de leur usage comme langue d'enseignement. Quant aux universités «anglophones», elles enseignent exclusivement en anglais, alors que les universités «francophones» enseignent en français. 
très facile et le français comme une langue difficile» (p. 88). Cette même idée a été sans cesse répétée par l'entourage de T. et W. «Chaque personne qui entend que je suis en train d'apprendre le français alors que je suis anglophone/ me dit/ msad'a halik/ you think you can do it $\uparrow$ même les francophones me disaient que le français était difficile pour eux»(W.). Les apprenants «francophones» comme les «anglophones» ont en fait le même état d'esprit : «les élèves qui apprennent l'anglais ont cette idéel que le français est difficile/ il vaut mieux choisir l'anglais / car il est plus facile/»(T.). Ces affirmations ne sont pas nouvelles, elles ne sont pas l'apanage d'une génération en particulier, mais continuent à circuler d'une génération à l'autre. C'est ainsi qu'en 1979, Rabbath déclare : «La maitrise du français est difficile en elle-même, à la différence de l'anglais, dont l'appréhension pourrait intervenir tardivement.» (p. XVII) Comme on l'a déjà remarqué, ces affirmations ne sont pas non plus le propre des non-spécialistes: «ma tante [elle-même professeure de français] m'avait dit si tu avais appris le français au début/ tu aurais été plus à l'aise dans l'apprentissage de l'anglais» (T.). D'ailleurs, Gueunier, Haddad et Aucagne (1993) avouent que «la première observation qui frappe, lors de l'analyse des entretiens, c'est la constante du motif de la "facilité" de l'anglais, opposé à celui de la "difficulté" du français» (p. 175). Cette image est renforcée par celle de la rigueur des professeurs de français «quand on fait une faute en français / le professeur va faire des remarques sur tous les détails sans exception / [...] alors que les profs d'anglais vont commenter les choses principales» (W.). Les résultats des examens officiels ne font que consolider cette image, puisque Hafez (2006) constate que, «le taux de réussite en français est très faible par rapport à l'anglais à tous les niveaux de l'apprentissage» (p. 108)

Y aurait-il vraiment des langues plus difficiles que d'autres? Qu'en est-il de cette représentation épistémique de ces deux langues?

Dans une enquête effectuée en France auprès de 300 étudiants et professeurs, Forlot (2009) note que $« 86 \%$ des personnes interrogées estiment qu'il existe, dans l'absolu, des langues plus difficiles que d'autres» (p. 16). D'ailleurs, la facilité constitue pour Dabène (1994) la deuxième des cinq échelles de mesure de ce qu'elle appelle le statut informel des langues formé d' «un ensemble de représentations fortement stéréotypées qui bien que fortement corrélées aux traditions idéologiques et culturelles en vigueur [...] sont, en général, fortement teintées de subjectivité» (p. 50). Cette image de la difficulté de la langue est pour Barbeiro (2013) «l'expression de la représentation de l'effort 
que le sujet doit faire pour arriver à comprendre ou à parler une langue particulière» (p. 151). Elle pourrait donc être dissuasive si les résultats obtenus dans l'apprentissage ne sont pas jugés dignes de l'effort fourni. Par contre, certains considèrent la facilité de la langue comme dévalorisante (Gueunier et coll., 1993, p. 177) et la difficulté comme valorisante et prestigieuse ${ }^{2}$.

Pour revenir à la langue française, Forlot (2009) signale dans son enquête précédemment citée que «83,5\% d'entre eux [300 étudiants et professeurs français] considèrent le français comme une langue difficile à apprendre pour un étranger» (p. 16). De même, Billiez (1996), dans une enquête réalisée dans le cadre du programme Galatea, cite les résultats de l'étude menée par Apotheloz et Bysaeth (1981) qui montre que les enquêtés neuchâtelois caractérisent la langue française comme «difficile», voire «inaccessible» (p. 406). De son côté, Barbeiro (2013) dans son enquête auprès d'élèves portugais a révélé que pour ces derniers «deux langues se détachent comme «difficiles»: le chinois et le français » (p. 152). Les Libanais ne sont donc pas les seuls à percevoir la langue française comme «difficile».

Dabène (1994) insiste sur «l'influence que peut avoir la représentation de facilité sur les stratégies déployées lors de l'apprentissage» (p. 51), mais aussi et surtout sur le choix de la langue à apprendre. Au Liban, le choix de l'école «francophone» à l'âge de trois ans est bien évidemment celui des parents et fait partie de la stratégie scolaire familiale (Bernaus et coll., 2003, p. 151). Mais le choix universitaire est sans doute soumis, au moins en partie, à la volonté du jeune universitaire. En effet, comme précédemment mentionné, de plus en plus de jeunes Libanais scolarisés dans des écoles «francophones» optent pour des universités «anglophones », considérées comme plus porteuses en terme d'avenir.

Il est intéressant de signaler que cette représentation de la langue anglaise en tant que langue facile et de la langue française comme difficile est tellement ancrée dans les esprits que nos deux étudiants W. et $\mathrm{T}$., qui ont par leur démarche infirmé l'idée très répandue quant à la difficulté de l'apprentissage du français après l'anglais, ont déclaré

2. C'est le cas de la langue allemande enseignée en France. Cette langue jugée difficile n'a été choisie pendant longtemps que par les meilleurs élèves, et également pour que les élèves soient placés dans des classes au niveau plus homogène, scolairement et socialement. 
qu'il «est plus facile d'apprendre le français après l'anglais / parce que tout le monde sait que l'anglais est plus simple»(W.). Ils ont donc fait usage du même argument «l'anglais est plus simple que le français» qui a toujours été utilisé pour convaincre les parents de scolariser leurs enfants dans des écoles francophones, et ce, dans le but de prouver le contraire : parce que l'anglais est plus simple, il est normal qu'on l'apprenne en premier. Il s'avère donc que, quel que soit l'ordre préconisé pour l'apprentissage des langues, le même argument de la difficulté du français et de la facilité de l'anglais est utilisé.

\subsection{Et aussi d'autres représentations de la langue française}

Cette représentation épistémique des langues, tout en étant l'une des plus marquées dans le contexte libanais, n'est néanmoins pas la seule. On constate que la langue française est de plus en plus cantonnée dans l'image culturelle, «le français c'est la culture» (W.), et plus particulièrement la culture littéraire «avec le français / j'ai pu apprécier la littératurel le français littéraire est à ne pas comparer avec l'anglais littéraire» (T.). C'est loin d'être nouveau dans la société libanaise, puisque Rabbath (1979) évoque «l'érection du français, langue de culture [dès l'époque de] l'empire ottoman» (p. XIv). Ce n'est pas non plus particulier aux locuteurs libanais. En effet, cette image du français comme langue de culture est très largement partagée dans de nombreux pays. C'est ainsi qu'au Maroc, Benzakour (2010) constate que le français «langue culturelle, $[\ldots]$ continue d'être le lieu d'une production littéraire importante» (p. 35). On retrouve la même situation en Pologne où «le français conserve toujours un caractère de luxe, de symbole de prestige, de culture» (Ziolkowski, 2004, p. 60). Il en est de même pour la Thessalie «la langue française était considérée dans la société thessalienne, tout comme aujourd'hui, une langue raffinée, utilisée par des couches sociales favorisées et porteuse d'un prestige de culture supérieure» (Kourdis, 2004, p. 39). D'ailleurs, dans son document Une langue pour apprendre, l'OIF (2010) après l'étude de l'«actualité de l'enseignement du et en français dans le monde» note que «l'image favorable dont elle [la langue française] jouit va traditionnellement de pair avec un certain prestige culturel» (p. 168). Cette image du français, véhicule d'une culture prestigieuse, est au demeurant limitative. En effet, le rôle attribué par les Libanais, des décennies durant, au français en tant que langue de la modernité et du lien avec le monde (Rabbath, 1979; Naaman, 1979; Abou, 1994 ; Haddad, 1994; Khorassandjian, 
1994; Tuéni, 1986), est actuellement rempli par l'anglais «c'est le link avec lequel je peux communiquer avec tout le monde/ avec cette langue je peux unlocker the whole world»(W.), ou alors, «avec l'anglais/ je suis devenu citoyen du monde» (T.). L'anglais est ainsi considéré comme la clé de l'ouverture au monde, une valeur sûre nécessaire pour une interaction à vaste échelle.

\section{Des représentations aux attitudes et aux réalités didactiques}

Ces images des langues circulent autant parmi les spécialistes que parmi les profanes et se répercutent directement sur les attitudes des apprenants face à l'apprentissage des langues au Liban. Selon Bernaus et coll. (2003) «les attitudes sont liées d'une part aux représentations des langues et des cultures et d'autre part aux stéréotypes associés aux locuteurs natifs de ces langues.» (p. 140). Dans les propos tenus dans les deux entretiens, représentations des langues et stéréotypes des locuteurs natifs cö̈ncident, «les Français ont leur histoire/ ils apprécient toutes les formes de l'art/ les Anglais apprécient aussi mais pas aussi bien que les Français/ ils ont un rapport plus strong avec les arts/ même le peuple français est plus cultivé artistiquement» (W.). Notons au passage la confusion absolue entre la langue française et la France et par suite l'exclusion de tout autre pays francophone.

\subsection{Attitude cruciale : la motivation}

Mais comment définir les attitudes? Selon Bernaus et coll. (2003), c'est la «disposition à [...] [l']état de préparation à un certain mode d'action» (p. 139). L'une des attitudes cruciales dans toute entreprise, y compris celle de l'apprentissage des langues, c'est la motivation (Candelier, 2003; Lauret, 2007; Viau, 2007). "L'attitude à l'égard de la langue étudiée est très importante : une motivation insuffisante à l'égard de celle-ci peut s'expliquer soit par une attitude ethnocentriste, soit par une antipathie véritable à l'égard de la langue étudiée.» (Debyser, 1970, p. 44) Dans le cas de la société libanaise plurilingue, on est loin de l'ethnocentrisme, mais bel et bien au cœur de cette «antipathie» grandissante.

Contrairement à T. qui n'avait jamais pris de cours de français car scolarisé dans une école syrienne où l'on apprend l'arabe et en arabe jusqu'à l'âge de six ans et où à partir de cet âge-là, l'anglais commence à être introduit comme seule langue étrangère, $\mathrm{W}$. avait fait trois ans de 
français troisième langue de l'âge de 10 à 12 ans, à raison d'une heure par semaine. W. certifie fermement l'inefficacité de cet apprentissage. La première raison du fait que les élèves apprennent mal le français dans la majorité des écoles «anglophones» libanaises proviendrait du manque de motivation lié au fait que les apprenants ne voient pas clairement la finalité de cet apprentissage jugé «inutile», «personne ne prenait la classe au sérieux/ parce qu'on n'avait pas besoin du français/»(W). Cette opinion est partagée par Diab (2006) :

La plupart des étudiants qui ont fréquenté des écoles anglophones ne prenaient pas au sérieux leurs classes de français et par conséquent ne consacraient pas beaucoup de temps et d'énergie à l'apprentissage de cette langue. Par contre, beaucoup d'étudiants diplômés d'écoles francophones croient qu'il est essentiel pour eux de bien connaitre l'anglais, une langue dont ils auraient vraisemblablement besoin à des fins académiques et professionnelles. (p. 89)

\subsection{Programmes d'enseignement "vieillots»}

Cette raison d'ordre pragmatique parait bien évidente, mais est-elle la seule responsable du manque de motivation pour l'apprentissage du français? La didacticienne Assaf Khoury (1998) avoue que, dans le contexte libanais, la langue française «souffre beaucoup au niveau de son apprentissage/enseignement [...] à cause de nos choix méthodologiques mal adaptés, de nos programmes [...] vieillots, de nos méthodes démodées et de nos manuels qui manquent d'intérêt» (p.48). Le manque de motivation est donc intrinsèquement lié aux programmes et aux modalités d'apprentissage du français. W. et T. le confirment pour certaines écoles «anglophones» : «La prof écrit au tableau / on apprend par cœur ce qu'elle écrit et on le note sur nos cahiers» (W.). Ces modalités d'apprentissage du français ne sont pas le propre des seules écoles «anglophones». Dans les écoles «francophones» aussi, l'enseignement de la langue française n'a pas bonne presse «les profs de français étaient tough with them [ses cousins et ses amis scolarisés dans des écoles francophones]/ Les profs d'anglais ne sont pas aussi sérieux»(W.).

D'un côté les «manuels ne répondent ni aux besoins ni aux ambitions des étudiants » (Chéhadé, 2008, p. 337), et d'un autre côté, les méthodes traditionnelles utilisées se résument par la pratique de la mémorisation et l'adoption des manuels comme seuls supports d'apprentissage, tout cela étant ajouté à l'intransigeance dans l'évaluation. Tous ces éléments participent à la démotivation des apprenants. Apparait aussi 
le handicap de la focalisation sur l'écrit (Assaf-Khoury, 1998; Chéhadé, 2008), puisque «l'enseignement du français ne s'occupe que du texte et de la langue écrite, ne recourant à l'oralité que pour la lecture à haute voix » (Chéhadé, 2008, p. 336). La négligence de l'oral à l'école, ajoutée à la rigueur des professeurs de français (soulignée ci-dessus par W.) pourrait figurer parmi les raisons principales pour lesquelles l'usage $\mathrm{du}$ français pour la communication quotidienne se ferait rare entre les jeunes selon T. : "à l'université de Balamand [à majorité anglophone] que ce soit pour discuter des cours / ou de la vie de tous les jours / on utilise un mélange d'arabe dialectal et d'anglais [...] même avec des personnes qui connaissent le français/ on parlait l'anglais/ parce que la majorité des étudiants qui avaient le français comme langue secondel avaient des problèmes à utiliser cette langue au quotidien/» (T.). Quand ce même étudiant a intégré l'Université Saint Joseph, «francophone» (désormais l'USJ) pour faire un Master en interprétation, il a constaté : «c'est plus le franbanais qui est utilisé. Ils utilisent des mots français dans des phrases arabes». Contrairement à ses attentes, T. constate la grande insécurité linguistique des francophones de l'USJ : rares sont ceux qui osent s'exprimer longuement en français. «[...] le statut de l'anglais à Balamand n'est pas le même que celui du français à l'USJ», l'anglais est utilisé plus aisément par les étudiants de Balamand que ne l'est le français par ceux de l'USJ. (T.) Cette constatation est confirmée par l'enquête de Hafez (2006) qui a conclu que pour les «anglophones» «l'écart est peu important entre l'anglais lu, compris, écrit ou parlé [alors qu'il l'était parmi les témoins francophones]. Il semblerait qu'ils ressentent moins d'insécurité linguistique que les francophones » (p. 182). Isabelle Bassil-Grappe (2010) insiste beaucoup sur cette insécurité linguistique en français des Libanais.

Toutes ces représentations du français et ces pratiques reliées à sa didactique au Liban sont loin de susciter l'engouement pour son apprentissage, même quand il s'agit d'un apprenant qui reçoit son éducation de base en français. Qu'en serait-il d'un anglophone?

En présentant les études supérieures en français comme inaccessibles après un apprentissage scolaire "anglophone», la population constituée des élèves «anglophones» est d'office exclue de cet enseignement. Quant aux élèves «francophones », ils sont divisés entre les deux catégories d'universités («anglophones» et «francophones»), ce qui aboutit à un déséquilibre quantitatif en faveur des universités «anglophones». Pour contrer cette vague et pour rassurer les apprenants (Hafez, 2006, p. 125), les universités libanaises «francophones» introduisent la langue 
anglaise dans leur enseignement. C'est ainsi que l'USJ, université «francophone» par excellence, a introduit l'anglais comme langue obligatoire $^{3}$ depuis 1999/2000. Alors que de leurs côtés, les universités «anglophones» ne ressentent pas la nécessité d'introduire la langue française dans leurs cursus.

Les représentations précédemment analysées de l'anglais et du français, en plus de l'absence de toute politique linguistique claire de la part du gouvernement libanais (Cuq, 1993; Hafez, 2006; Eid, 2012) ont sûrement leurs parts de responsabilité dans cette pratique universitaire.

Le non-dirigisme linguistique étant l'une des constantes de l'État libanais, démontrer, dans le cadre de la formation initiale et continue des enseignants, à l'aide de données objectives et d'analyses des représentations, la fausseté de la croyance qui consiste à présenter le français comme inaccessible aux «anglophones», travailler les modalités d'enseignement du français dans le système scolaire libanais en général et dans les écoles «anglophones» en particulier, aideront à ouvrir les portes du trilinguisme et accessoirement celles des études supérieures en français aux élèves «anglophones» au même titre qu'à leurs collègues «francophones».

Les deux étudiants $\mathrm{W}$. et $\mathrm{T}$. ont prouvé par leur démarche intuitive et individuelle défiant les préjugés que le trilinguisme des anglophones libanais n'est pas un mirage, mais une réalité accessible à tout anglophone qui le désire.

\section{Conclusion}

En somme, les représentations de la langue française en tant que langue «difficile» qui n'est pas vraiment «utile» pour les études d'abord et l'emploi plus tard, ont une influence directe sur les attitudes des apprenants libanais vis-à-vis de son apprentissage et de sa pratique au quotidien. Mais ce qu'il ne faudrait jamais perdre de vue c'est que «les attitudes sont construites, il faut savoir comment elles peuvent être changées» (Bernaus et coll., 2003, p. 140). Il en est de même pour le stéréotype, «on le croit à tort fixé», mais il est «toujours en mouvement» (Goulet, 1994, p. 7). Nous avons très clairement constaté à travers l'expérience de W. et de T. qu'il n'en était rien de la prétendue

3. Pour plus d'informations, voir Le français langue obligatoire à l'USJ, <www. usj.edu.lb/admission/anglais.htm> (consulté le 15 août 2014). 
relation de cause à effet : le français est difficile, l'anglais est facile, donc l'apprentissage du français après l'anglais est impossible. Ces deux «anglophones» ont réussi à apprendre le français à l'âge adulte et à intégrer une filière de traduction. S'ils ont pu le faire, d'autres le peuvent aussi à condition qu'ils soient suffisamment éclairés et motivés. Il convient d'ailleurs de revenir, dans cette conclusion, sur le point de départ de cette relation, c'est-à-dire l'idée de la difficulté intrinsèque de certaines langues, ce qui est contesté par tous les didacticiens, comme l'affirme Assaf-Khoury (1998) : «La langue française [...] n'est pas plus difficile à apprendre que d'autres langues.» (p. 45) C'est ainsi que les problèmes rencontrés dans l'apprentissage d'une langue ne relèvent pas de «la complexité de son système linguistique [...] mais surtout $[\ldots]$ de nos choix méthodologiques mal adaptés, de nos programmes [...] vieillots, de nos méthodes démodées et de nos manuels qui manquent d'intérêt» (Assaf-Khoury, 1998, p. 48). La mission principale des didacticiens est donc d'œuvrer pour la modernisation des manuels de français, et ce en les rendant plus attractifs et interactifs, centrés autant sur l'oral que sur l'écrit. D'autre part, il serait pertinent de réfléchir dans toutes les écoles libanaises à «la formation à l'“arbitraire esthétique" de la langue» (Forlot, 2009, p. 17). Si cet «arbitraire esthétique» des langues est impossible à atteindre, il faudrait au moins ne pas adopter une attitude fataliste et défaitiste, mais œuvrer d'abord à l'extériorisation de ces représentations, leur analyse et leur comparaison avec les données objectives. Le fait de cantonner la langue française dans le domaine culturel, même si nous le considérons dans une perspective plus large en admettant que «les réalités culturelles informent les réalités politiques» (Maïla, 2007, p. 66) risque de l'exclure du monde de la communication internationale en général, et scientifique/technique en particulier.

Il est souhaitable que ce travail de conscientisation et d'essai de remodelage des représentations soit fait en premier lieu auprès des enseignants et plus particulièrement les enseignants de langue française qui ont une grande part de responsabilité non seulement dans la diffusion de ces représentations, mais aussi dans l'enracinement de ces dernières à travers certaines pratiques didactiques : mémorisation, centration sur l'écrit, attitude puriste et perfectionniste qui met les apprenants d'emblée dans une situation d'impuissance, d'insécurité linguistique, et par suite d'échec. L'une des démarches à suivre serait celle de rendre, à l'image du Liban, l'enseignement des langues plurilingue. Non dans le sens d'accumuler les enseignements indépendants de trois langues 
dans le même cursus, comme c'est le cas aujourd'hui, mais dans celui d'assurer un dialogue entre les langues enseignées. Ce dialogue interrogera constamment les acquis langagiers des apprenants dans les autres langues et en profitera pour un apprentissage plus efficace et plus économique en termes de temps et d'efforts.

\section{RÉFÉRENCES BIBLIOGRAPHIQUES}

ABou, Sélim \& Haddad, Katia. (1994). Une francophonie différentielle. Paris : L'Harmattan.

Abou, Sélim, Kasparian, Choghig \& Haddad, Katia. (1996). Anatomie de la francophonie libanaise. Beyrouth : FMA.

Aвou, Sélim. (2008). L'avenir du français au Machrek. Dans J. Maurais et coll. (dir.), L'avenir du français (p. 201-204). Paris : Éditions des archives contemporaines, AUF.

Ambassade de France au Liban. (2011). Coopération linguistique et édu-

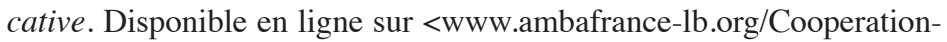
linguistique-et> (consulté le 24 avril 2014).

Apotheloz, Denis \& Bysaeth, Léo. (1981). Attitudes linguistiques : résultats d'une enquête. Tranel, 2, 69-90.

Assaf Khoury, Carméline. (1998). Didactique du français langue étrangère/seconde : pour un renouveau méthodologique de l'enseignement/ apprentissage du français au Liban (Thèse de doctorat non publiée). Université Nancy 2.

Barbeiro, Luis Filipe. (2013). Parcours pour l'éducation linguistique plurilingue : représentation et découverte des langues. Synergies Portugal, $1,145-162$.

BASSIL-GrapPe, Isabelle. (2010). Conception et évaluation d'une Formation de formateurs au français sur objectifs spécifiques à partir d'une approche sociolinguistique (Thèse de doctorat non publiée). Université Stendhal-Grenoble 3, Grenoble.

Benzakkour, Fouzia. (2010). Le français au Maroc, enjeux et réalité. Le français en Afrique, 25, 33-41, <http://www.unice.fr/ILF-CNRS/ofcaf/ 25/Table\%20des\%20matieres.pdf>.

Bernaus, Merce, Genelot, Sophie, Hensinger, Caroline \& Matthey Marinette. (2003). Evlang et la construction des attitudes. Dans M. Candelier (dir.), L'éveil aux langues à l'école primaire. Evlang: bilan d'une innovation européenne (p. 139-155). Bruxelles : De Boeck.

BILLIEZ, Jacqueline. (1996). Langues de soi, langues voisines : représentations entrecroisées. Études de linguistique appliquée, 104, 401-410. 
Candelier, Michel (dir). (2003). L'éveil aux langues à l'école primaire. Bruxelles : De Boeck.

ChéHadé, Claudia. (2008). Le Liban, Pivot de la francophonie au MoyenOrient (Thèse de doctorat non publiée). Université Bordeaux 3.

CuQ, Jean-Pierre. (1993). Le français langue seconde, origines d'une notion et implications didactiques. Paris : Hachette.

CuQ, Jean-Pierre \& Davin-Chnane, Fatima. (2007). Français langue seconde : un concept victime de son succès. Dans M. Verdelhan-Bourgade (dir.), Le français langue seconde : un concept et des pratiques en évaluation (p. 11-28). Bruxelles : De Boeck.

DABÈnE, Louise. (1975). L'enseignement de l'espagnol aux francophones (pour une didactique des langues «voisines»). Langages, 39, 51-64.

DABÈNE, Louise. (1994). Repères sociolinguistiques pour l'enseignement des langues. Paris: Hachette.

DABÈnE, Louise. (1997). L'image des langues et leur apprentissage. Dans M. Matthey (dir.), Les langues et leurs images (p. 19-23). Neuchâtel : IRDP.

Debyser, François. (1970). La linguistique contrastive et les interférences. Langue française, 8, 31-61.

Diab, Rula. (2006). University Students' Beliefs about Learning English and French in Lebanon. System: An International Journal of Educational Technology and Applied Linguistics, 34(1), 80-96.

EID, Cynthia. (2012). L'enseignement du Français sur Objectifs Spécifiques / Français de Spécialisation à l'Université : approche globale d'une discipline, perspective professionnelle et universitaire? Dialogos, 13, 67-88.

Forlot, Gilles. (2009). Vers la déconstruction d'un apprentissage idéologique. Des «représentations-obstacles» dans l'enseignement des langues en France. Les cahiers de l'Acedle, 6(1), 69-91.

Goulet, Alain. (1994). Avant-propos. Dans Le stéréotype. Crises et transformations. Actes du colloque du Cerisy-La-Salle (p. 7-8). Caen : Presses universitaires de Caen.

Gueunier, Nicole, Haddad, Katia \& Aucagne, Jean. (1993). Le français au Liban, cent portraits linguistiques. Paris : Didier Érudition.

HAFEz, Stéphane-Ahmad. (2006). Statuts, emplois, fonctions, rôles et représentations du français au Liban. Paris : L'Harmattan.

HADDAD, Katia. (2002). La francophonie dans le monde arabe : état des lieux, Beyrouth : Agence universitaire de la francophonie.

Hoteit, Sanaa. (2010). Enseignement-apprentissage du français au sud du Liban : didactique contextualisée et intégration dans une dynamique culturelle francophone (Thèse de doctorat non publiée). Université Rennes 2. 
HoYeK, Samir. (2004). Le français dans l'enseignement scolaire et universitaire au Liban. Cahiers de l'Association internationale des études françaises, 56, 49-56.

KouRDIs, Evangelos. (2004). La langue française dans les Balkans. Étude sociolinguistique des emprunts français dans le dialecte de Thessalie (Grèce centrale). Langue et Littérature romanes, Annales de l'université Craiova, numéro spécial, 38-44.

LAURET, Bertrand. (2007). Enseigner la prononciation du français : questions et outils. Paris : Hachette.

Maïla, Joseph. (2007). Quels processus démocratiques en Francophonie? Dans K. Haddad (dir.), La francophonie aujourd'hui et demain (p. 6573). Beyrouth : Presses de l'Université Saint-Joseph.

NAAman, Abdallah. (1979). Le français au Liban. Beyrouth : Naaman.

Rabbath, Edmond. (1979). Préface. Dans A. Naaman, Le français au Liban. Beyrouth : Naaman.

SMAÏlY-HAJJAR, Wajiha. (1996). Le français et l'anglais langues étrangères au Liban: analyse de leurs statuts actuels (Thèse de doctorat non publiée). Université Nancy 2.

TuÉNI, Nadia. (1986). La prose : æeuvre complète. Beyrouth : Dar an-nahar.

Université SAINT Joseph, Le français langue obligatoire à l'USJ. Disponible en ligne sur <www.usj.edu.lb/admission/anglais.htm> (consulté le 15 août 2014).

VIAU, Rolland. (2007). La motivation en contexte scolaire. Bruxelles : De Boeck.

Zıolkovski, Marek. (2004). La francophonie en Pologne. Hermes, 40, 59-61. 